\title{
Variable-Speed Induction Motor Drives for Aircraft Environmental Control Compressors
}

J.W. Mildice, I.G. Hansen, and K.E. Schreiner Motive Power Development

San Diego, California

M.E. Roth

Lewis Research Center

Cleveland, Ohio

Prepared for the

31st Intersociety Energy Conversion Engineering Conference cosponsored by IEEE, AIChE, ANS, SAE, AIAA, and ASME Washington, D.C., August 11-16, 1996

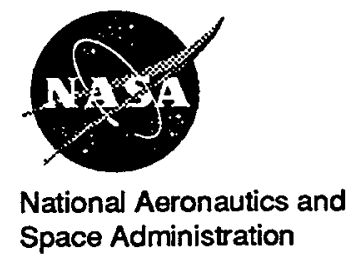




\title{
VARIABLE-SPEED INDUCTION MOTOR DRIVES FOR AIRCRAFT ENVIRONMENTAL CONTROL COMPRESSORS
}

\author{
J. W. Mildice; I. G. Hansen; K. E. Schreiner \\ Motive Power Development \\ 7905 Silverton Avenue, Suite 109 \\ San Diego, California 92126 \\ Telephone (619) 536-5556; FAX (619) 536-5558 \\ M. E. Roth \\ NASA LeRC, Mail Stop 301-2 \\ 21000 Brookpark Road \\ Cleveland, Ohio 44135 \\ Telephone (216) 433-8061; FAX (216) 433-2469
}

\begin{abstract}
New, more-efficient designs for aircraft jet engines are not capable of supplying the large quantities of bleed air necessary to provide pressurization and air conditioning for the environmental control systems (ECS) of the next generation of large passenger aircraft. System analysis and engineering have determined that electrically-driven ECS can help to maintain the improved fuel efficiencies; and electronic controllers and induction motors are now being developed in a NASA/MPD SBIR Program to drive both types of ECS compressors.

An important operational requirement for these applications is variable speed/load operation; and total maximum power outputs are under $200-\mathrm{kW}$ for cabin pressurization and $100-\mathrm{kW}$ for vapor cycle compressors, divided among four redundant units in a typical, 300 passenger, wide-body design. Typical speeds for full output of aircraft centrifugal compressors are in the 50,000-RPM range. This performance is now within reach for modern, resonant-technology, power processors and for improved induction motors. This technology implementation offers significant improvements over the more typical, switched-mode controllers and "brushless DC" motor designs.

Previous variable-speed induction motor/controller system developments and publications have primarily focused on field-oriented control, with large transient reserve power, for maximum acceleration and optimum response in actuator and robotics systems. The application area addressed herein is characterized by slowly-changing inputs and outputs, small reserve power capability for acceleration, and optimization for maximum efficiency.

This paper therefore focuses on the differences between this case and the optimum response case, and shows the development of this new motor/controller approach. It
\end{abstract}

starts with the creation of a new set of controller requirements. In response to those requirements, new control algorithms are being developed and implemented in an embedded computer, which is integrated into the motor controller closed loop. Buffered logic outputs are used to drive the power switches in a resonant-technology, power processor/ motor-controller, at switching/resonant frequencies high enough to support efficient high-frequency induction motor operation at speeds up to 50,000 -RPM.

\section{AIRCRAFT SYSTEM CONFIGURATION}

For the purposes of this research, the electrical power system configuration chosen is the one under development for the Power-by-Wire (PBW) testbed demonstration, currently sponsored and directed by NASA LeRC. The target aircraft for that configuration was chosen to be a 300 passenger, wide-bodied, twin engine model, in the MD-11 class.

The electrical system for that program is supplied from four variable-speed, engine-driven alternators (two on each engine). Modular architecture opportunities and redundancy issues led to a system configuration with four separate, variable-frequency, three-phase alternator output busses connected to four electrical power conditioning modules. These modules provide fixed-frequency, voltage-controlled, $400-\mathrm{Hz}$ power for the aircraft systems.

Changes in demand with altitude and outside temperature, above-normal operation to compensate for a failed unit, and overall efficiency issues demand variable-speed capability for the compressor motor drives. This capability requires a power processor to interface between the $\mathrm{AC}$ power system and the compressor drive motor, independent of the technology chosen. For the maximum load case, 
electrical power for ECS operation is more than half of the total on-board power requirement. Since the motor control power processors can operate with a variable-frequency $\mathrm{AC}$ input as easily as a fixed-frequency one, it was decided to power the ECS System directly from the three-phase, variable-frequency, engine alternator output busses rather than the $400-\mathrm{Hz}$ conditioned, aircraft distribution busses. This reduces the power output requirements for the $400-\mathrm{Hz}$ utility power processors and provides for a significant end-to-end system efficiency improvement.

\section{SPECIFIC ECS HARDWARE REQUIREMENTS}

Maintaining the "quad" distribution approach, there are then four ECS modules, each providing a motor drive and compressor for the vapor cycle cooling system and cabin air pressurization. Cabin pressurization requires about twice the output power as the vapor cycle compressors. For demonstration and test purposes, we have chosen to use the cabin pressurization application. The primary requirements for the cabin pressurization compressors in each of the four modules are presented in Table 1.

\section{Table 1, Drive Requirements (per ECS module)

$\begin{array}{ll}\text { Speed } & \\ \text { Normal Operation }= & 48,900 \mathrm{RPM} \\ \text { One Compressor Out }= & 54,344 \mathrm{RPM} \\ \text { Mass Flow Rate } & \\ \quad \text { Normal Operation }= & 0.88 \mathrm{lb} / \mathrm{sec} \\ \quad \text { One Compressor Out }= & 0.88 \mathrm{lb} / \mathrm{sec} \\ \text { Pressure Ratio } & \\ \quad \text { Normal Operation }= & 3.54 \\ \quad \text { One Compressor Out }= & 3.54 \\ \text { Aero Efficiency } & \\ \quad \text { Normal Operation }= & 0.80 \\ \quad \text { One Compressor Out }= & 0.76 \\ \text { Motor Shaft Power } \\ \text { Normal Operation }= & 61.4 \text { Horsepower } \\ \text { One Compressor Out }= & 100.4 \text { Horsepower }\end{array}$

To promote modularity and common hardware designs, we have elected to use $30 / 50-\mathrm{kW}$ motor and drive electronics modules for both applications. The vapor cycle compressor uses one; and cabin pressurization uses a tandem/ dual motor drive system with torque summing.

The drive motor choice arguments have continued in the aerospace community for many years, with advocates for induction motors or permanent magnet motors seldom convincing one another. Therefore, we will not start another round of that discussion here. However, induction motors have clearly won the debate for industrial and consumer applications. In general, they are the most commonly-used motor (in all sizes) throughout the world, and are cheaper, simpler, and more reliable than the other available choices. Because of their easy variable-speed control, the strong commercial legacy required of SBIR programs of this type, and in the spirit of modern government COTS programs, we have selected induction motors as the prime movers for the two compressor applications.

There is no such series of discussions about the power processor/motor controller choice for this power capability. Because of their improved efficiency and reduced power switch stresses, resonant power processors are the clear choice.

\section{MOTOR DESIGN}

The preferred class of centrifugal compressors for both of these applications typically operate with maximum speeds in the 50,000-RPM range. While smaller permanent magnet motors with contact-type bearings have approached this speed, and non-contact bearing designs have reached the $100,000-R P M$ range, induction motors have generally not been constructed for these speeds. But there is no inherent induction motor unique speed limit, if high-speed technologies similar to those used in permanent magnet motors are applied to induction motors.

Therefore, a new three-phase, squirrel-cage, induction motor has been designed for this program, which incorporates high-speed contact-type bearings, integral speed sensing, and a proprietary rotor bar containment system. A cross-sectional diagram of that motor is shown in Figure 1.

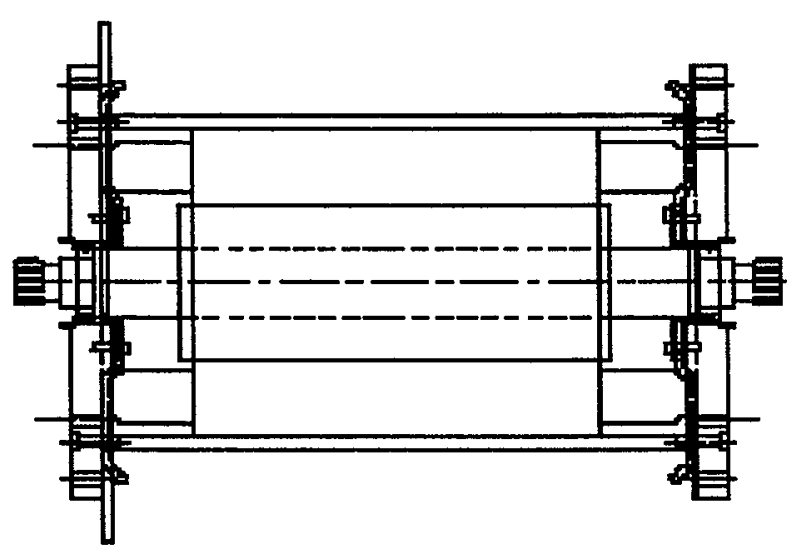

Figure 1, High-Speed Induction Motor

Since torque summing is easy to control for induction motors, this design is stackable, to provide for a building block in a modular drive system which can be easily expanded for higher power outputs. As described earlier, a pair of stacked motor modules are used to drive the cabin pressurization compressor for this development and demonstration. Motor physical characteristics and performance are documented in Table 2. 
Table 2, Induction Motor Characteristics

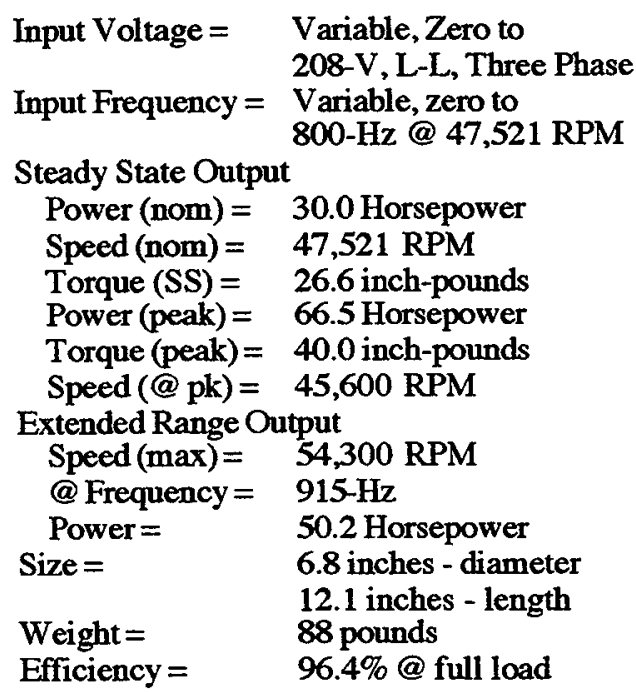

\section{MOTOR CONTROL POWER PROCESSOR} DESIGN

The input power for these motor controllers is the direct output from a variable-frequency, engine-driven alternator. Specifications are listed in Table 3 . Since energy recovery and bi-directional power flow is not required for the ECS application, we have complete flexibility for the power source interface, and simple rectifier inputs are acceptable, as long as power quality requirements can be met.

Even though it is not in the normal aircraft operating range of frequencies, $60-\mathrm{Hz}$ was added to the input power requirements to enable operation from standard utility power sources. This addition is only a minimal design complication, and it simplifies laboratory and ground test operations, and provides an easier transition to commercial and consumer hardware; which is an important issue for Phase 3 of SBIR programs of this type.

For actuators, the transient reserve power for acceleration is typically equal to or greater than the steady-state power requirement to move the load at its maximum rate. For example, a well-designed 50-horsepower actuator would require a minimum of a 100 -horsepower power processor and motor just to overcome the system inertias for acceptable acceleration and deceleration. Drive systems (except perhaps for one used in a Drag Racer) can operate with less than $10 \%$ reserve power, and the power processor and motor capabilities can be reduced accordingly. So a 50-horsepower vehicle-class drive system is about half as big as a 50-horsepower motor drive for a large actuator.

A set of model-based, trade studies were performed which evaluated switched-mode controllers, activelyclamped, DC-link (voltage-source) resonant power processors, actively-clamped series-resonant (current-source) reso- nant power processors, and adjustable pulse width series-resonant (current-source) resonant power processors. The performance parameters traded were efficiency, output ripple, overall stability, size, and weight. We have chosen a DC link, resonant power processor with a three-phase output bridge, which interfaces directly with the drive motor. This topology has the highest efficiency of all the primary candidates, and is capable of operating at the highest frequency for this power level (in the $75-\mathrm{kHz}$ range). This enables smaller filters (with reduced input and output ripple) and higher output frequency response for better control stability. Also, with the proper output filter design, its motor current ripple is almost as good as the current source power processor (which had the lowest ripple in the trades).

As you can see from Table 2, nominal motor operation requires a 30 -horsepower output. If we consider motor losses and power factor, we need a power processor output of about 30-KVA. Applying the same factors, the extended-range, system failure-compensation mode will require about $50-K V A$. If we allow an additional $10 \%$ acceleration power, the maximum capability of the power processor output gets to about 55-KVA. The output voltage is variable, controlled by the resonant converter PPM, and consistent with the maximum 208-vAC, RMS phase voltage for the motor.

\section{Table 3, Input Power Characteristics}

\begin{tabular}{|c|c|}
\hline $\begin{array}{c}\text { Input Voltage }= \\
\text { (operational) }\end{array}$ & $\begin{array}{l}115 / 208-\mathrm{V}, \mathrm{RMS} \pm 10 \% \\
\text { Three Phase } \\
\text { or } \\
210 \text { - to } 238-\mathrm{V}, \mathrm{RMS}, \mathrm{L-N} \\
\text { (three phase average) } \\
\text { 206- to } 245-\mathrm{V}, \mathrm{RMS}, \mathrm{L-N} \\
\text { (individual phase voltage) } \\
\text { Three Phase }\end{array}$ \\
\hline $\begin{array}{l}\text { Input Voltage } \\
\text { (abnormal) }\end{array}$ & $\begin{array}{l}\text { 205- to 248-V, RMS, L-N } \\
\text { (three phase average) } \\
190 \text { - to } 252-\mathrm{V}, \mathrm{RMS}, \mathrm{L-N} \\
\text { (individual phase voltage) } \\
\text { Three Phase }\end{array}$ \\
\hline Input Frequency = & $\begin{array}{l}60-\mathrm{Hz} \\
\text { or } \\
\text { Variable, 365- to } 995\end{array}$ \\
\hline $\mathrm{THD}=$ & $8 \%$ of the fundamental \\
\hline
\end{tabular}

If we were designing this controller for a vehicle drive system with maximum cruise requirements about the same as the steady state for this program, and used this motor as a modular building block, it could provide very high performance. We could design the controller for as much as 65-KVA and have the capability for very good acceleration, by using the peak torque capability of the basic motor design. 
MOTOR CONTROL ALGORITHM DEVELOPMENT

Actuator development for aerospace applications and robotics has understandably focused on optimum-response or critically-damped systems. They have traditionally been implemented with field-oriented-control computer algorithms when induction motors are used instead of permanent magnet (DC brushless) motors. The class of "drive" applications addressed by this research, which includes both compressor and vehicle drive systems, may be characterized as speed controlled, slowly-responding, optimum-efficiency systems.

Implementation of a motor speed control loop is straight-forward, whether the primary command is generated by a human foot on a "throttle" or a command from an ECS computer. For induction motors, the relationship between nominal input voltage and input frequency is linear between about $10 \%$ and $100 \%$ speed. So the speed command can also be used to determine the nominal applied voltage. But optimizing efficiency demands adjustment of motor voltage and/or current to match changing motor parameters or changing system load.

If we look at efficiency indicators which do not require sampling and testing to measure the optimum input power point, those which show the highest correlation are motor power factor and slip. (See Figure 2 for the slip characteristic of a typical high-efficiency induction motor.)

Slip Limit Efficiency

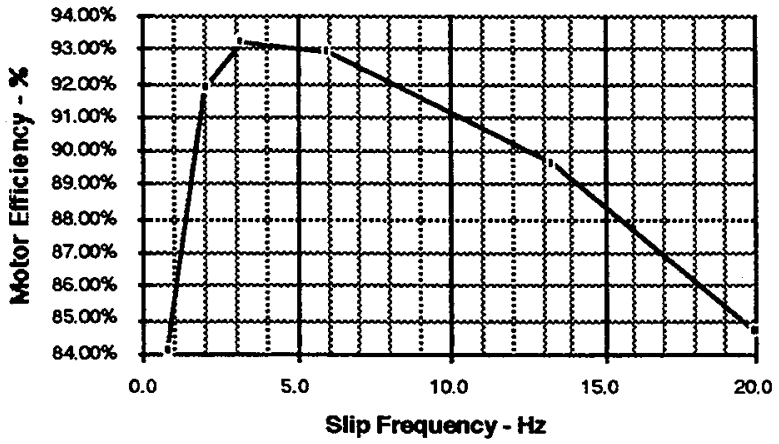

Figure 2, High-Efficiency Induction Motor Efficiency Correlations

Power factor is difficult to measure in pulse or resonant power processors because of the output voltage or current ripple. The slip is easy to determine, but the magnitude required for a particular load changes as the motor rotor resistance changes with operating temperature. But as you can see from Figure 2, efficiency stays near its maximum over a reasonably broad range of slip. This suggests that we can use a simple control system that regulates slip to an average value, with only a small compromise in our selection of optimum efficiency (approximately $0.3 \%$ for this motor). For example, the motor of Figure 2 operates close to its best efficiency when the slip frequency is between $3-\mathrm{Hz}$ and 6-Hz. Using this approach, we developed a primary control system which is shown in Figure 3.

An input speed command generates internal motor frequency and voltage commands, based on the nominal V/f ratio for full-load operation. A slip control loop, which responds to a slip reference input, modifies the V/f ratio so that it is appropriate to the actual motor load. For slow responding systems, this is all that is required. For faster response (as in vehicle drives), an acceleration input raises the V/f ratio and allows a larger slip to provide increased output torque.

\section{Enhanced Fuzzy State Selection}

One important output requirement for switched PWM or resonant PDM power processors in this application is output ripple. Most present PDM designs compare the instantaneous output voltage or current in each motor phase with an intemally-generated reference, and decide whether or not to output the next pulse. A "state selector" then determines the on-off output pattern for the six power switches in the three-phase output bridge driving the motor.

We have determined that output ripple can be improved if we add additional parameters to the instantaneous error signal threshold normally used. These are voltage amplitude, voltage polarity, slope of the voltage waveform, commanded (or inferred) acceleration, and load conditions.

For example (using only two of the parameters), if the output error is very large, we would always apply a correcting pulse. But if the output error is in a mid-range, we might or might-not apply a correcting pulse, depending on the polarity of the error and the slope of the reference sine wave. When we consider one or two of the other parameters in the same way, the decision process quickly begins to sound like the definition of a set of fuzzy rules. And in fact, we have designed a fuzzy-type hardware implementation to output a Phase X (+) pulse, Phase X (-) pulse, of Phase X (no) pulse decision. are:

Some examples of the fuzzy rules used in this design

If Amplitude Error (A) is "large (+),"

then switch $\mathrm{A}(-)=\mathrm{ON}$ and switch $A(+)=$ OFF

If Amplitude Error (A) is "large (-)," then switch $\mathrm{A}(+)=\mathrm{ON}$ and switch $A(-)=$ OFF

If Amplitude Error (A) is "medium (+)," and Slope $(A)$ is "large (-) and V/f value (A) is "large," then switch $A(-)=O N$ and switch $A(t)=\mathrm{OFF}$

If Amplitude Error (A) is "medium," and Slope $(A)$ is "large $(+)$ and V/f value $(A)$ is "medium,"

then switch $\mathrm{A}(-)=\mathrm{OFF}$ and switch $\mathrm{A}(+)=\mathrm{OFF}$

If ... 


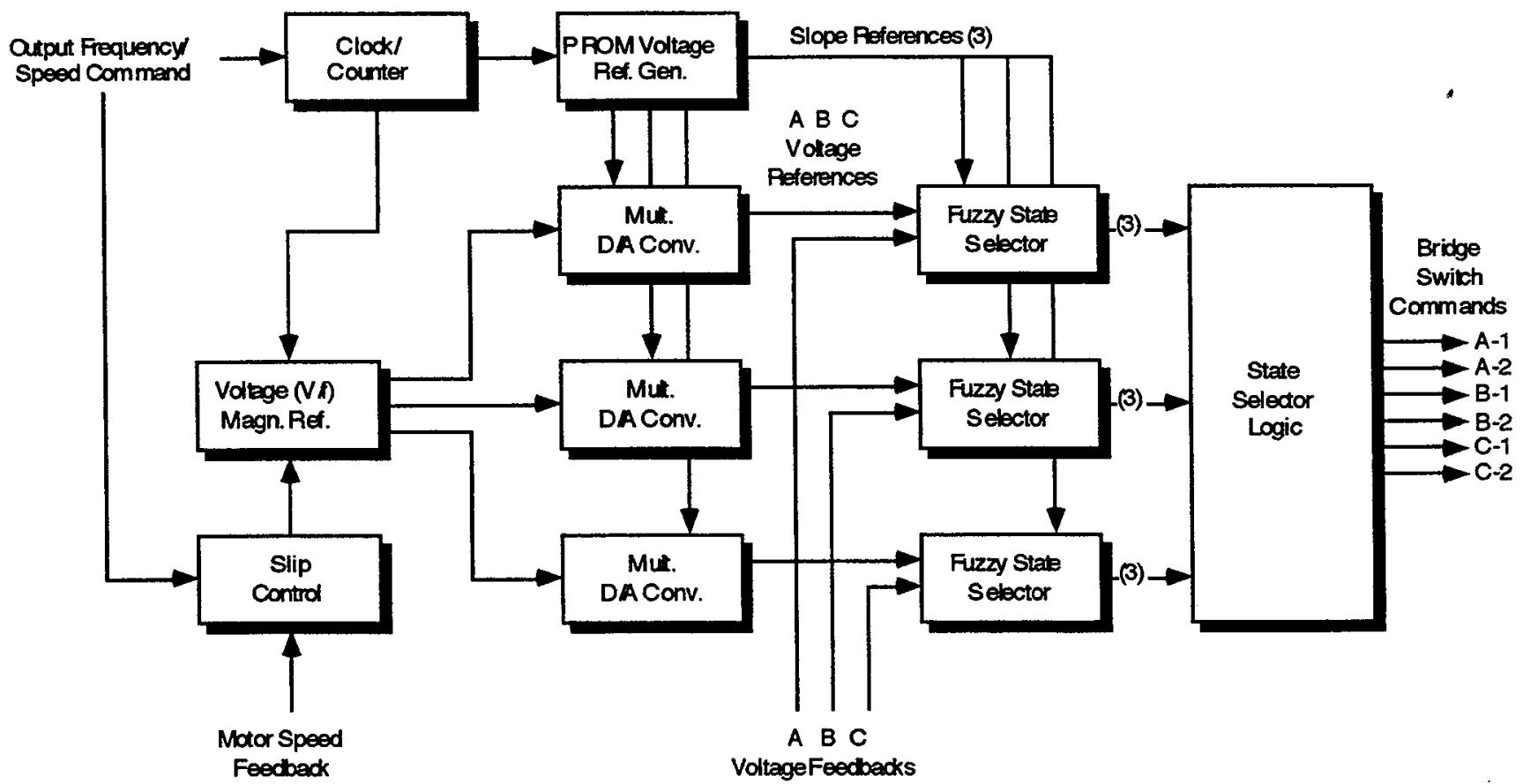

Figure 3, Controller Block Diagram

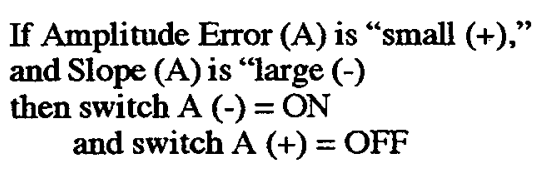

If ...

Because of space limitations, we will not list the complete set of fuzzy rules and membership functions here, but the fuzzy sets used in this controller are:

- Amplitude Error, (magnitude and polarity).

- V/f Value (also provides load inference).

- Slope of the commanded sine wave.

- Polarity of the commanded sine wave.

- Accelerationrequirement

Since the control inputs and feedback start out as analog quantities, and because of the nature of the control loop, we are performing the fuzzy set combinations in a hardware-based amplifier configuration. A simplified diagram of this implementation is shown in Figure 4. The number of amplifiers has been minimized through superposition, and some of the quantities (such as amplitude error) seem to disappear in the actual schematic.

Individual quantity gains control the intersecting slopes of the membership functions. "Defuzzification" is performed by the output threshold function, and the outputs enable the individual commands for the power switches in the three-phase bridge connected to the motor windings.

There is one such loop for each of the motor windings, so the output commands are Phase $\mathrm{X}$ high (+ voltage), low (ground), and no connection, for each of the three motor phases. This allows us to command an increase, decrease, or no change the individual motor phase voltages.

The output logic block provides for additional safety and output enhancement functions. It prevents simultaneous $\mathrm{ON}$ states in a single phase, to eliminate the possibility "shoot-through" failures for errors or transients. It also inhibits a limited set of sequential output states which could cause large reverse motor current transients.

PPM output changes are synchronized to the resonant frequency of the power processor. The switch states have the opportunity to be changed at each cycle, and the state commands generated by the fuzzy state selectors are clocked into an output register for the next output switch cycle. In this way a decision can be made for the modification of the motor applied inputs on a pulse-by-pulse basis, for the high-frequency power processor carrier frequency. Motor ripple current is reduced by the motor inductance and an LC output filter, for the output three-phase power provided to the motor. 


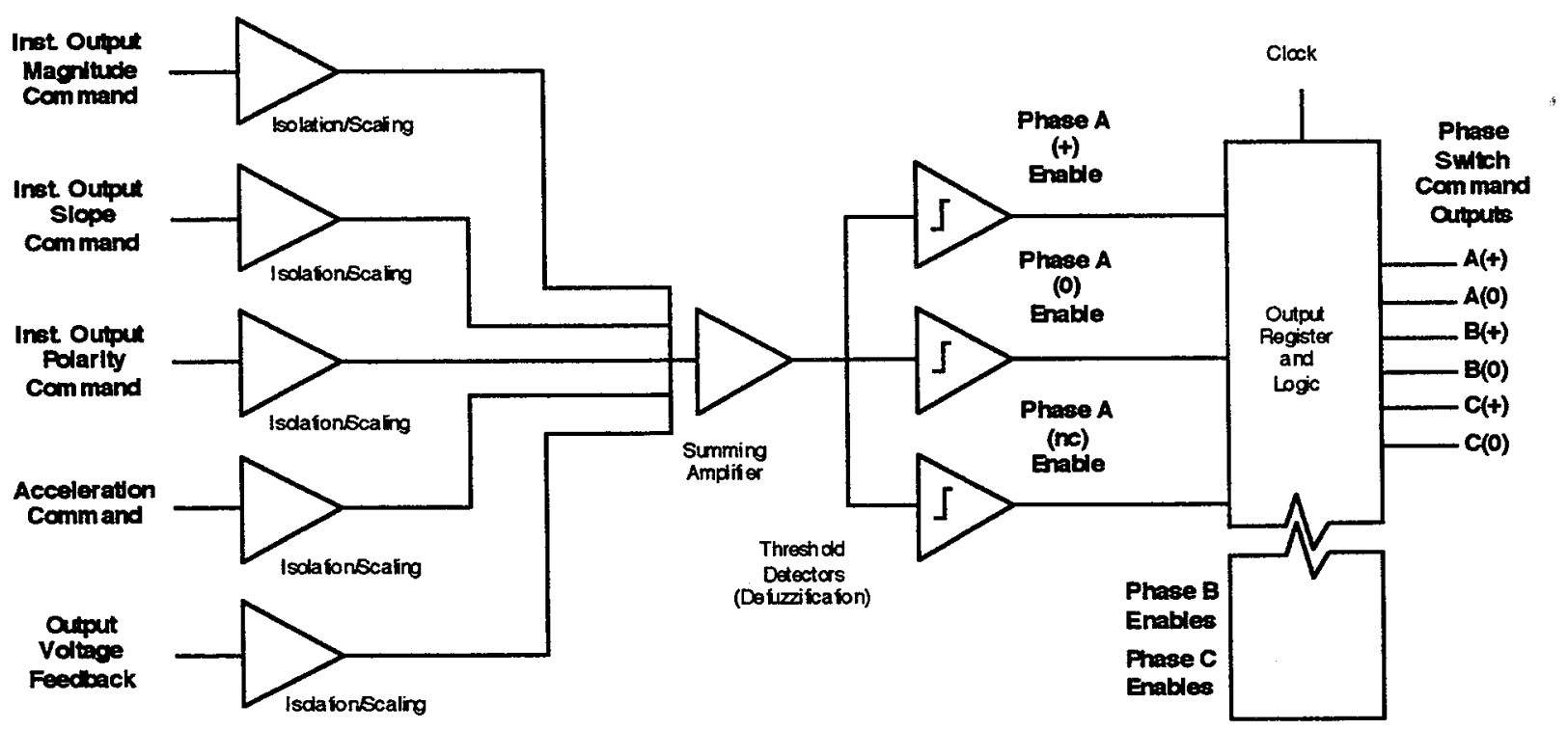

Figure 4, Enhanced, Fuzzy Type State Selector for Each Phase

\section{CONCLUSIONS}

This report documents a research program performed by Motive Power Development for NASA LeRC on a Phase 2 SBIR contract. As such, its primary goal is to provide technology development in support of NASA interests and programs, as this does. This motor drive system is an integral part of the technology necessary for the development of more efficient, environmentally-compatible commercial aircraft. At the same time, there is a strong interest in commercial "spin-offs."

This aircraft ECS design can be simply extrapolated into the commercial and consumer sector. The motor drives can be used (without much change) for large facility environmental control when those systems are new or upgraded designs which use the new, environmentally-compatible, non-CFC refrigerants.

Because of their modular capabilities and low losses, the motor controllers can be coupled with lower speed induction motors, and are therefore also compatible with high-efficiency drives for both large and small electric vehicles.

The high-speed induction motors will enable new terrestrial systems which will contain a new class of high speed, commercial, centrifugal compressors to replace the piston types now in use, for higher efficiency and lower maintenance.

This program is not yet complete. We are reporting on these developments at the point where design and analysis are complete, and we are building the first sets of test hardware. Therefore, there will be a follow-up report to document the results of the testing, through the installation and test in an actual ECS compressor system. 
Public reporting burden for this collection of information bs estimated to average 1 hour per response, inchuding the time for reviewing instructions, searching extsting data sources, gathering and maintaining the data needed, and completing and reviowing the colloction of information. Send commonis regarding this burden estons and Reports, 1215 Jefferson

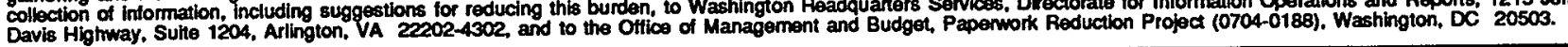

\begin{tabular}{l|l|l} 
1. AGENCY USE ONLY (Leave blank) & $\begin{array}{c}\text { 2. AEPORT DATE } \\
\text { July } 1996\end{array}$ & $\begin{array}{c}\text { 3. REPORT TYPE AND DATES COVERED } \\
\text { Technical Memorandum }\end{array}$
\end{tabular}

\section{TITLE AND SUBTITLE}

Variable-Speed Induction Motor Drives for Aircraft Environmental Control Compressors

6. AUTHOR(S)
5. FUNDING NUMBERS

WU-244-02-02

J.W. Mildice, I.G. Hansen, K.E. Schreiner, and M.E. Roth

7. PERFORMING ORGANIZATION NAME(S) AND ADDRESS(ES)

National Aeronautics and Space Administration

Lewis Research Center

Cleveland, Ohio 44135-3191
8. PERForming ORGANIZATION REPORT NUMBER

E-10325

9. SPONSORINGMONITORING AGENCY NAME(S) AND ADDRESS(ES)

National Aeronautics and Space Administration

Washington, D.C. 20546-0001

10. SPONSORINGMONTTORING AGENCY REPORT NUMBER

NASA TM-107261

\section{SUPPLEMENTARY NOTES}

Prepared for the 31st Intersociety Energy Conversion Engineering Conference, cosponsored by IEEE, AIChE, ANS, SAE, AIAA, and ASME, Washington, D.C., August 11-16, 1996. J.W. Mildice, I.G. Hansen, and K.E. Schreiner, Motive Power Development, 7905 Silverton Avenue, Suite 109, San Diego, California 92126 (work funded by NASA Contract NAS327549); M E. Roth, NASA Lewis Research Center. Responsible person, M.E. Roth, organization code 5430, (216) 4338061.

128. DISTRIBUTIONAVAILABILTY STATEMENT 12b. DISTRIBUTION CODE

\section{Unclassified - Unlimited}

Subject Categories 01 and 33

This publication is available from the NASA Center for AeroSpace Information, (301) 621-0390.

13. ABSTRACT (Maximum 200 words)

New, more-efficient designs for aircraft jet engines are not capable of supplying the large quantities of bleed air necessary to provide pressurization and air conditioning for the environmental control systems (ECS) of the next generation of large passenger aircraft. System analysis and engineering have determined that electrically-driven ECS can help to maintain the improved fuel efficiencies; and electronic controllers and induction motors are now being developed in a NASA/MPD SBIR Program to drive both types of ECS compressors. Previous variable-speed induction motor/controller system developments and publications have primarily focused on field-oriented control, with large transient reserve power, for maximum acceleration and optimum response in actuator and robotics systems. The application area addressed herein is characterized by slowly-changing inputs and outputs, small reserve power capability for acceleration, and optimization for maximum efficiency. This paper therefore focuses on the differences between this case and the optimum response case, and shows the development of this new motor/controller approach. It starts with the creation of a new set of controller requirements. In response to those requirements, new control algorithms are being developed and implemented in an embedded computer, which is integrated into the motor controller closed loop. Buffered logic outputs are used to drive the power switches in a resonant-technology, power processor/motor-controller, at switching/resonant frequencies high enough to support efficient high-frequency induction motor operation at speeds up to 50,000-RPM.

14. SUBJECT TERMS

Induction motors; Power converters; Environmental control; Fuzzy systems; Aircraft; Cabin atmospheres; Resonant frequencies

15. NUMBER OF PAGES 08 16. PRICE CODE $\mathrm{AO} 2$

\begin{tabular}{l|c|}
\hline $\begin{array}{c}\text { 17. SECURTY CLASSIFICATION } \\
\text { OF REPORT } \\
\text { Unclassified }\end{array}$ & $\begin{array}{c}\text { 18. SECUATY CLASSIFICATION } \\
\text { OF THIS PAGE } \\
\text { Unclassified }\end{array}$ \\
\hline
\end{tabular}

19. SECURITY CLASSIFICATION OF ABSTRACT Unclassified 
National Aeronautics and

Space Administration

\section{Lewis Research Center}

21000 Brookpark Rd.

Cleveland, $\mathrm{OH}$ 44135-3191

Official Business

Penalty for Private Use $\$ 300$

POSTMASTER: If Undeliverable - Do Not Return 